\title{
Quadrupole moments of spherical semi-magic nuclei within the self-consistent Theory of Finite Fermi Systems
}

\author{
S. V. Tolokonnikov \\ Kurchatov Institute, 123182 Moscow and \\ Moscow Institute of Physics and Technology, 123098 Moscow, Russia \\ S. Kamerdzhiev and D. Voitenkov \\ Institute for Physics and Power Engineering, 249033 Obninsk, Russia \\ S. Krewald \\ Institut für Kernphysik, Forschungszentrum Jülich, D-52425 Jülich, Germany \\ E. E. Saperstein \\ Kurchatov Institute, 123182 Moscow
}

\begin{abstract}
The quadrupole moments of odd neighbors of semi-magic lead and tin isotopes and $N=50, N=$ 82 isotones are calculated within the self-consistent Theory of Finite Fermi Systems based on the Energy Density Functional by Fayans et al. Two sets of parameters, DF3 and DF3-a, fixed previously are used. They differ by the spin-orbit and effective tensor force parameters, the latter being significantly bigger in the DF3-a functional. Results for the two functionals turned out to be rather different. The functional DF3-a leads to quadrupole moments in reasonable agreement with the experimental ones for most nuclei examined.
\end{abstract}

PACS numbers: 21.10.-k, 21.10.Jx, 21.10.Re, 21.60-n

\section{INTRODUCTION}

In the last decade, the interest for ground state nuclear properties, in particular, static moments, was renewed due to modern Radioactive Ion Beam facilities which provide access to long chains of isotopes, including the radioactive ones, in their ground and isomeric states. Such nuclei distant from the $\beta$-decay stability valley and often very close to the drip lines are of great interest for nuclear astrophysics. Spectroscopy techniques using high intensity lasers allow for precision measurements of nuclear spins, magnetic and quadrupole moments which are much more delicate nuclear characteristics than masses and charge radii. As a result, the bulk of the data on nuclear static moments becomes very extensive and comprehensive [1] creating a challenge to nuclear theory. Recent precise measurements of magnetic and quadrupole moments of a long chain of copper isotopes from $N=28$ to $N=46$ presented and analyzed in the comprehensive article 2] is a new evidence of this interest. For nuclear magnetic moments, this challenge was partially responded recently [3, 4]. Data on magnetic moments of more than one hundred odd nuclei were reproduced with an accuracy of $0.1-0.2 \mu_{\mathrm{N}}$ within the self-consistent Theory of Finite Fermi Systems (TFFS). In this study, the QRPA-like TFFS equations for the nuclear response to the external magnetic field were solved on the base of the Energy Density Functional (EDF) by Fayans et al. [5-7]. Especially high accuracy was reached for semimagic nuclei considered in the "single-quasiparticle approximation" where one quasiparticle in the fixed state $\lambda=(n, l, j, m)$ with the energy $\varepsilon_{\lambda}$ is added to the even- even core. Such nuclei where only one kind of nucleons is superfluid are, as a rule, spherical. In addition, the lowest $2^{+}$-state has usually an excitation energy of the order $1 \mathrm{MeV}$, the corresponding collectivity is not extremely high and the particle-phonon coupling is not sufficiently strong to destroy the single-quasiparticle approximation. According to the TFFS [8], a quasiparticle differs from a particle of the single-particle model in two respects. First, it possesses a local charge $e_{q}$ and, second, the core is polarized due to the interaction between the particle and the core nucleons via the Landau-Migdal (LM) amplitude. In other words, the quasiparticle possesses the effective charge $e_{\text {eff }}$ caused by the polarizability of the core which is found by solving the TFFS equations. In the many-particle Shell Model [9], a similar quantity is introduced as a phenomenological parameter which describes polarizability of the core consisting of outside nucleons. For nuclei with both non-magic subsystems corrections to the single-quasiparticle approximation due to $2^{+}$-phonon coupling are important and should be taken into account to describe magnetic moments correctly [3, 4] .

For quadrupole moments, the systematic calculations we know concern only the medium atomic weight nuclei of the $2 p-1 f$ shell [9] and $2 p, 1 f_{5 / 2}, 1 g_{9 / 2}$ shell [2]. Evidently, the first step in this direction for heavy nuclei was made in our recent article [10] where we analyzed effects of the density dependence by studying the $2_{1}^{+}$-excitations in two isotopic chains of semi-magic even-even tin and lead nuclei within a version of the self-consistent TFFS which is very similar to that in $[3,4]$. The only difference is the use of a new version DF3-a [11] of the original DF3 
functional [6, 7]. It differs from DF3 only by the spinorbit parameters $\varkappa, \varkappa^{\prime}$ and the first harmonics $g_{1}, g_{1}^{\prime}$ of the spin LM amplitude. The corresponding force components determine mainly the spin-orbit doublet splitting and are of especial importance for high $j$-levels. The last two terms modify the spin-orbit component of the mean field together with the spin-orbit density $\rho_{s l}(r)$ which is determined with particles of partially filled spin-orbit doublets. As it is well known, the first spin harmonics contribute to the mean field in a combination with tensor forces, see e.g. 12]. Therefore, they should be considered as the effective first harmonic or, on equal footing, the effective tensor force. It should be mentioned that in both the functionals DF3 and DF3-a the parameter $g_{1}$ is taken equal to zero, thus, the spin-orbit splitting is determined with the set of three parameters, $\varkappa, \varkappa^{\prime}, g_{1}^{\prime}$. For brevity, we will name them just spin-orbit. The functional DF3-a is characterized by a rather strong effective tensor force.

In this paper we concentrate on quadrupole moments. We limit ourselves to semi-magic nuclei, where the onequasiparticle approximation is expected to provide a good accuracy. In addition to two isotopic chains considered in [10], we calculate quadrupole moments of odd neighbors of even isotones with $N=50$ and $N=82$ and of four odd neighbors of the magic nucleus ${ }^{40} \mathrm{Ca}$. We compare predictions of two functionals, DF3 and DF3-a, which have different spin-orbit components. It is important for the problem under consideration as quadrupole moments $Q_{\lambda}$ are proportional to the Bogolyubov factor [13]:

$$
u_{\lambda}^{2}-v_{\lambda}^{2}=\left(\varepsilon_{\lambda}-\mu\right) / E_{\lambda},
$$

where $E_{\lambda}=\sqrt{\left(\varepsilon_{\lambda}-\mu\right)^{2}+\Delta_{\lambda}^{2}}$, with obvious notation. In the quadrupole moments problem we deal with the ground state of an odd nucleus or with very low-lying excited state when often the inequality $\left|\varepsilon_{\lambda}-\mu\right| \ll \Delta_{\lambda}$ is true. In such a situation, the quadrupole moment value is very sensitive to the single-particle energy $\varepsilon_{\lambda}$. In its turn, the latter is very sensitive to the spin-orbit parameters of the EDF. This could help in fixing these parameters not known sufficiently well up to now. The reason is that nuclear masses and radii used mainly for finding effective force parameters are not-sensitive to such parameters as $\kappa^{\prime}, g_{1}, g_{1}^{\prime}$. Note that the relevance of the spin-orbit and effective tensor force to other low-energy nuclear phenomena was discussed recently in Refs. [14 16]. In this paper, we consider only the surface kind of pairing as motivated by previous research of Refs. [7, 16, 17].

\section{BRIEF CALCULATION SCHEME}

The calculation scheme of the self-consistent TFFS based on the EDF method by Fayans et al. is described in detail in Ref. [10]. Here we summarize several formulas which are necessary for understanding the main ingredients of the approach. The EDF method by Fayans et al. [5-7] is a generalization for superfluid systems of the original Kohn-Sham EDF method [18]. For condensed matter case, similar generalization was carried out in Ref. [19]. In this method, the ground state energy of a nucleus is considered as a functional of normal and anomalous densities,

$$
E_{0}=\int \mathcal{E}\left[\rho_{n}(\mathbf{r}), \rho_{p}(\mathbf{r}), \nu_{n}(\mathbf{r}), \nu_{p}(\mathbf{r})\right] d^{3} r .
$$

Within the TFFS, the static quadrupole moment $Q_{\lambda}$ of an odd nucleus with the odd nucleon in the state $\lambda$ can be found in terms of the diagonal matrix element $\langle\lambda|V(\omega=0)| \lambda\rangle$ of the effective field $V$ in the static external field $V_{0}=\sqrt{16 \pi / 5} r^{2} Y_{20}$. In systems with pairing correlations, equation for the effective field can be written in a compact form as

$$
\hat{V}(\omega)=e_{q} \hat{V}_{0}(\omega)+\hat{\mathcal{F}} \hat{A}(\omega) \hat{V}(\omega),
$$

where $e_{q}$ is the local quasiparticle charge with respect to the external field $V_{0}$ and all other terms are matrices. In the standard TFFS notation [8], we have:

$$
\begin{gathered}
\hat{V}=\left(\begin{array}{c}
V \\
d_{1} \\
d_{2}
\end{array}\right), \quad \hat{V}_{0}=\left(\begin{array}{c}
V_{0} \\
0 \\
0
\end{array}\right), \\
\hat{\mathcal{F}}=\left(\begin{array}{ccc}
\mathcal{F} & \mathcal{F}^{\omega \xi} & \mathcal{F}^{\omega \xi} \\
\mathcal{F}^{\xi \omega} & \mathcal{F}^{\xi} & \mathcal{F}^{\xi \omega} \\
\mathcal{F}^{\xi \omega} & \mathcal{F}^{\xi \omega} & \mathcal{F}^{\xi}
\end{array}\right), \\
\hat{A}(\omega)=\left(\begin{array}{ccc}
\mathcal{L}(\omega) & \mathcal{M}_{1}(\omega) & \mathcal{M}_{2}(\omega) \\
\mathcal{O}(\omega) & -\mathcal{N}_{1}(\omega) & \mathcal{N}_{2}(\omega) \\
\mathcal{O}(-\omega) & -\mathcal{N}_{1}(-\omega) & \mathcal{N}_{2}(-\omega)
\end{array}\right),
\end{gathered}
$$

where $\mathcal{L}, \mathcal{M}_{1}$, and so on stand for integrals over $\varepsilon$ of the products of different combinations of the Green function $G(\varepsilon)$ and two Gor'kov functios $F^{(1)}(\varepsilon)$ and $F^{(2)}(\varepsilon)$. They can be found in [8]. In our case, the local charges in Eq. (3) are $e_{q}^{p}=1, e_{q}^{n}=0$.

Isotopic indices in Eqs. (446) are omitted for brevity. The explicit form of the above equations is written down for the case of the electric ( $t$-even) symmetry we deal with. In Eq. (5), $\mathcal{F}$ is the usual LM amplitude,

$$
\mathcal{F}=\frac{\delta^{2} \mathcal{E}}{\delta \rho^{2}},
$$

$\mathcal{F}^{\xi}$ is the density-dependent effective pairing interaction,

$$
\mathcal{F}^{\xi}(\rho)=\frac{\delta^{2} \mathcal{E}}{\delta \nu^{2}},
$$

and the amplitudes $\mathcal{F}^{\omega \xi}=\mathcal{F}^{\xi \omega}$ stand for the mixed second derivatives,

$$
\mathcal{F}^{\omega \xi}=\frac{\delta^{2} \mathcal{E}}{\delta \rho \delta \nu} .
$$


In the case of volume pairing, one has $\mathcal{F}^{\omega \xi}=0$, whereas for the case of surface pairing we deal the amplitude $\mathcal{F}^{\omega \xi}$ is non-zero and should be taken into account when Eqs. (446) are solved. As the analysis of Ref. [10] shows, the component $V$ of the vector $\hat{V}$, as a rule, dominates. However, the fields $d_{1}, d_{2}$ also contribute, and sometimes significantly, to the value of $Q_{\lambda}$.

The final expression for the quadrupole moment of an odd nucleus is as follows [8, 13]

$$
Q_{\lambda}^{p, n}=\left(u_{\lambda}^{2}-v_{\lambda}^{2}\right) V_{\lambda}^{p, n},
$$

where $u_{\lambda}, v_{\lambda}$ are the Bogolyubov coefficients and

$$
V_{\lambda}=-\frac{2 j-1}{2 j+2} \int V(r) R_{n l j}^{2}(r) r^{2} d r .
$$

It should be noted that we use here, just as in Ref. [10], the diagonal in $\lambda$ approximation for the gap, $\Delta_{\lambda, \lambda^{\prime}}=$ $\Delta_{\lambda} \delta_{\lambda, \lambda^{\prime}}$ where the Bogolyubov set of equations is reduced to the BCS scheme. In the general case, the relation for the quadrupole moment similar to Eq. (10) is more complicated. The $j$-dependent factor in (11) appears due to the angular integral [20]. For $j>1 / 2$ it is always negative. For odd neighbors of a magic nucleus the "Bogolyubov" factor in (10) reduces to 1 for a particle state and to -1 for a hole one. If the odd nucleon belongs to the superfluid component, the factor $\left(u_{\lambda}^{2}-v_{\lambda}^{2}\right)$ in Eq. (10) becomes non-trivial, see Eq. (11). It changes permanently depending on the state $\lambda$ and the nucleus under consideration. Note that in the case of magnetic moments the factor of $\left(u_{\lambda}^{2}+v_{\lambda}^{2}\right)=1$ appears [13] in the relation analogous to (10). In our case, this factor determines the sign of the quadrupole moment. It depends essentially on values of the single-particle basis energies $\varepsilon_{\lambda}$ reckoned from the chemical potential $\mu$ as. Keeping in mind such sensitivity, we found this quantity for a given odd nucleus $(Z, N+1)$ or $(Z+1, N), N, Z$ even, with taking into account the blocking effect in the pairing problem [13] putting the odd nucleon to the state $\lambda$ under consideration. For the $V_{\lambda}$ value in Eq. (10) for superfluid nuclei, we used the half-sum of these values in two neighboring even nuclei.

\section{CALCULATION RESULTS}

The set of nuclei analyzed includes, in addition to odd neighbors of two chains of even isotopes of tin $(Z=50)$ and lead $(Z=82)$ considered in [10], odd neighbors of even isotones with $N=50$ and $N=82$. To check the method for lighter nuclei, we calculated also quadrupole moments of odd neighbors of the magic ${ }^{40} \mathrm{Ca}$ nucleus. We concentrate on one of the two versions of pairing interaction considered in [10], the surface one, as it was favored by that investigation. On the other hand, we consider two versions of the EDF. In addition to the DF3-a functional used in [10] we made alternative calculations with the original DF3 functional of [6, 7]. All parameters of the two functionals are given in [10].

Let us begin by analysing nuclei with odd neutron numbers. Quadrupole moments of the odd Sn isotopes are displayed in Fig 1. Predictions for the two versions of the functional, DF3 and DF3-a with different spin-orbit parameters, are compared with each other and with the experimental data. In average, the results of both versions reasonably agree with the data. Each theoretical curve crosses the zero line due to vanishing of the corresponding Bogolyubov factor in Eq. (10). The analogous experimental curve could be approximately displayed only for the $11 / 2^{-}$-state. We see that it crosses the zero close to both theoretical curves, the difference between those is $\delta A \simeq 2$.

Before analysing the results for both versions of the EDF in detail, it is instructive to discuss the $A$ dependence of the position of the state $1 h_{11 / 2}$ and compare it with the experimental one. For the tin chain, this "intruder" state plays a special role due to the high $j$ value and closeness to the Fermi level. Indeed, the propagator $\mathcal{L}(\omega=0)$ of Eq. (6) playing the main role in the TFFS equation for the effective field $\hat{V}_{2+}(\omega=0)$ contains diagonal elements $\mathcal{L}_{\lambda \lambda}=-\Delta_{\lambda}^{2} /\left(2 E_{\lambda}^{3}\right)$. Their contribution is proportional to the $\left(2 j_{\lambda}+1\right)$ factor. If the energy $E_{\lambda}\left(h_{11 / 2}\right)$ is small, the contribution of this level to $\mathcal{L}$ can be rather big. Therefore a change in the position of this level may change the result for the quadrupole moment significantly.

As it was explained above, the DF3 and DF3-a functionals differ with spin-orbit parameters only, the difference being important mainly for high $j$-levels. The $1 h_{11 / 2}$ level position accounted from the ground state in Sn isotopes, $E_{\lambda}-\mu$, for both the functionals for even tin isotopes is displayed in Fig. 2 in comparison with the experimental excitation spectrum in neighboring odd nuclei. The corresponding values of the Bogolyubov factor $u_{\lambda}^{2}-v_{\lambda}^{2}$ are shown in the right-up sector of the figure. Dealing with an even-even ${ }^{A} \mathrm{X}$ nucleus, say, even ${ }^{A} \mathrm{Sn}$ isotope, there is a dilemma how to interpret a state $|\lambda\rangle$ under consideration, either the hole state (i.e. the excitation of the ${ }^{A-1} \mathrm{Sn}$ nucleus) or the particle one (the excitation of the ${ }^{A+1} \mathrm{Sn}$ nucleus). We use a simple ansatz: the state $|i\rangle$ is considered as a hole state if the inequality $\left(u_{\lambda}^{2}-v_{\lambda}^{2}\right)<0$ takes place and as a particle state otherwise. Note that in the case of $\left(u_{\lambda}^{2}-v_{\lambda}^{2}\right) \simeq 0$ the difference between the particle and hole energies is, as a rule, quite small. Fortunately, for the main part of the chain there is no contradiction between both the functionals in the "particle/hole" interpretation of the $1 h_{11 / 2}$ state. In the left part, up to ${ }^{120} \mathrm{Sn}$ nucleus, we deal with particle states and, correspondingly, for each $A$ value here the green triangle shows the excitation energy of the $(A+1)$ th isotope. On the right side, $A \geq 120$ experimental data show the ground state $11 / 2^{-}$, or almost ground state in ${ }^{130,132} \mathrm{Sn}$ isotopes. Therefore, a formal contradiction in this point for the ${ }^{122} \mathrm{Sn}$ doesn't work. We see that both functionals predict $A$-dependence of the excitation en- 


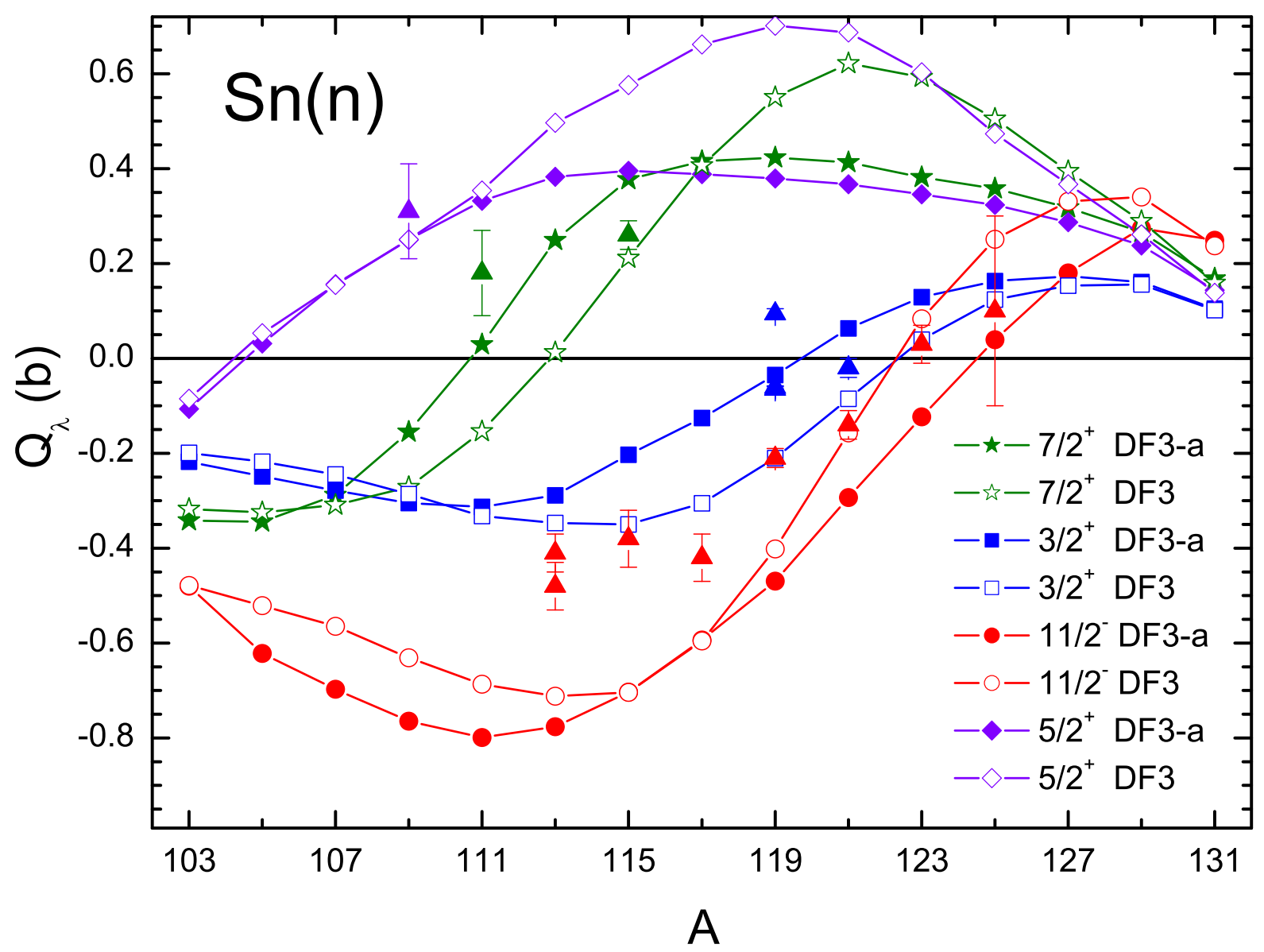

FIG. 1: (Color online) Quadrupole moments of odd tin isotopes with DF3 and DF3-a functionals. Triangles with the bars indicate experimental data [1].

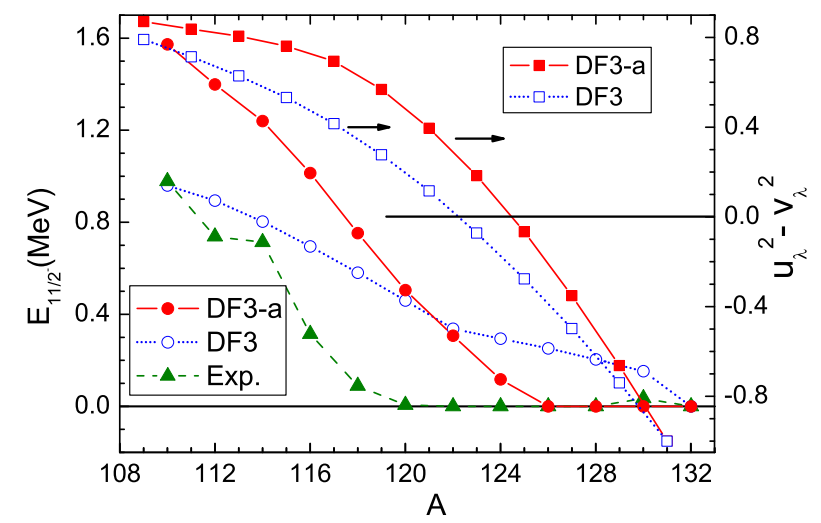

FIG. 2: (Color online) For the $h_{11 / 2}$ level in Sn isotopes: the energy $E_{\lambda}$ accounted from the ground state(the left-down legend; left axis) and values of the Bogolyubov $\left(u_{\lambda}^{2}-v_{\lambda}^{2}\right)$ factor (right-up legend; right axis).

ergy of the $h_{11 / 2}$ level qualitatively similar to the experimental one, falling in the value with $A$ increasing, but both theoretical curves are higher than the experimental

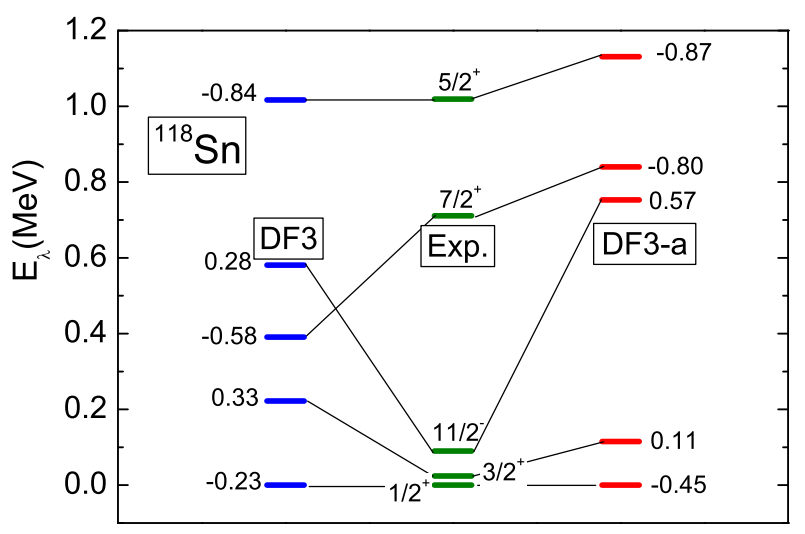

FIG. 3: (Color online) Single-particle spectrum of the ${ }^{118} \mathrm{Sn}$ nucleus. Value of the Bogolyubov $\left(u_{\lambda}^{2}-v_{\lambda}^{2}\right)$ factor is given near each theoretical crossbar.

one. Beginning from $A=124$, the DF3-a points practically coincide with the experimental ones. The DF3-a curve is above the DF3 curve to the left of the crossing point between $A=120$ and $A=122$, to the right, the 


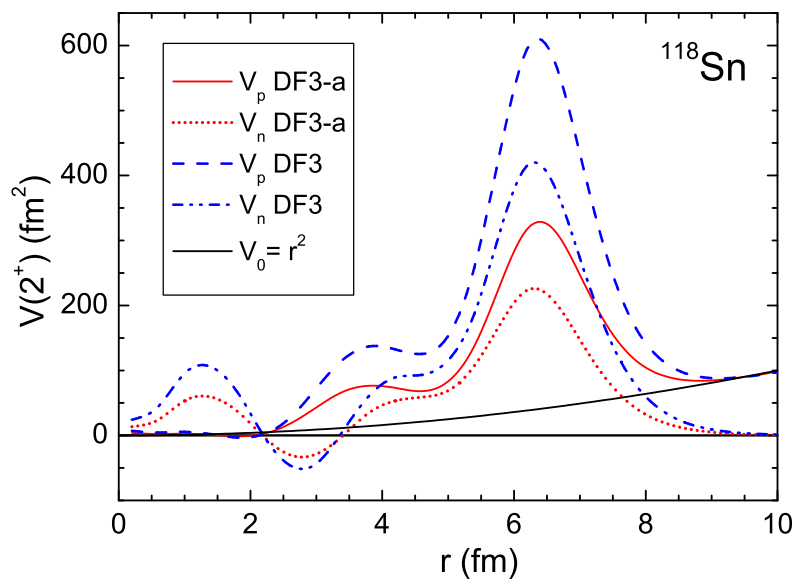

FIG. 4: (Color online) Effective field $V(r)$ in ${ }^{118} \mathrm{Sn}$ nucleus with DF3 and DF3-a functionals.

position is inverse. For almost all the $A$ values the inequality $\left(u_{\lambda}^{2}-v_{\lambda}^{2}\right)_{\mathrm{DF} 3}<\left(u_{\lambda}^{2}-v_{\lambda}^{2}\right)_{\mathrm{DF} 3-\mathrm{a}}$ is valid. Absolute values of the Bogolyubov factor for these functionals differ often significantly, as a rule, more than the quadrupole moment values, see Fig. [1 and Table [I] Analysis shows that it occurs due to the opposite effect in the matrix elements $V_{\lambda}$ of the effective field.

Let us analyze this point in more detail for the nucleus ${ }^{118} \mathrm{Sn}$ which is in the middle of the tin chain. The excitation spectrum for this nucleus is displayed in Fig. 3 . According to the ansatz formulated above, position of experimental levels for $3 / 2^{+}, 11 / 2^{-}$states are taken from the spectrum of the ${ }^{119} \mathrm{Sn}$ nucleus, that of $7 / 2^{+}, 5 / 2^{+}$ states, from ${ }^{117} \mathrm{Sn}$ isotope. Values of the Bogolyubov $u_{\lambda}^{2}-v_{\lambda}^{2}$ are also calculated in corresponding odd nuclei with the blocking effect taken into account as described in Chapter 2. In this nucleus positions of $11 / 2^{-}$state for both functionals are rather close to each other as this nucleus is in the vicinity of the crossing point in Fig. 2 However energies of the $7 / 2^{+}$state, also with rather big momentum value, are strongly different. On the whole, the density of states at the Fermi surface for the DF3 functional is higher than for DF3-a one. This difference is, in fact, significant if one takes into account the factor of $(2 j+1)$ with which each $j$-state comes to this quantity. To avoid misleading, we note that the comparison of the theoretical spectrum in the even-even $A$-nucleus which is the basis for the QRPA-like calculation with the experimental excitation spectra of neighboring odd nuclei, as is made in Figs. 213, is, in general, rather approximate operation. In heavy nuclei, corrections to the mean field single-particle spectra appear due to particlephonon coupling. Phenomenological functionals, as DF3 or DF3-a, or any SHF functional, take into account such corrections only on average. For quantitative description of single-particle spectra, all fluctuations, from one nucleus to another and from one state to another, should be calculated explicitly.
TABLE I: Characteristics of single-particle states in ${ }^{118} \mathrm{Sn}$ nucleus.

\begin{tabular}{ccccccc}
\hline \hline$\lambda$ & $u_{\lambda}^{2}-v_{\lambda}^{2}$ & $V_{\lambda}$ & $Q_{\lambda}$ & $u_{\lambda}^{2}-v_{\lambda}^{2}$ & $V_{\lambda}$ & $Q_{\lambda}$ \\
\hline \multicolumn{5}{c}{ DF3 } & & \multicolumn{5}{c}{ DF3-a } \\
$2 d_{5 / 2}$ & -0.84 & -0.838 & +0.704 & -0.87 & -0.449 & +0.390 \\
$1 g_{7 / 2}$ & -0.58 & -0.954 & +0.553 & -0.80 & -0.549 & +0.439 \\
$1 h_{11 / 2}$ & 0.28 & -1.454 & -0.407 & 0.57 & -0.826 & -0.471 \\
$2 d_{3 / 2}$ & 0.33 & -0.636 & -0.210 & 0.11 & -0.341 & -0.038 \\
\hline \hline
\end{tabular}

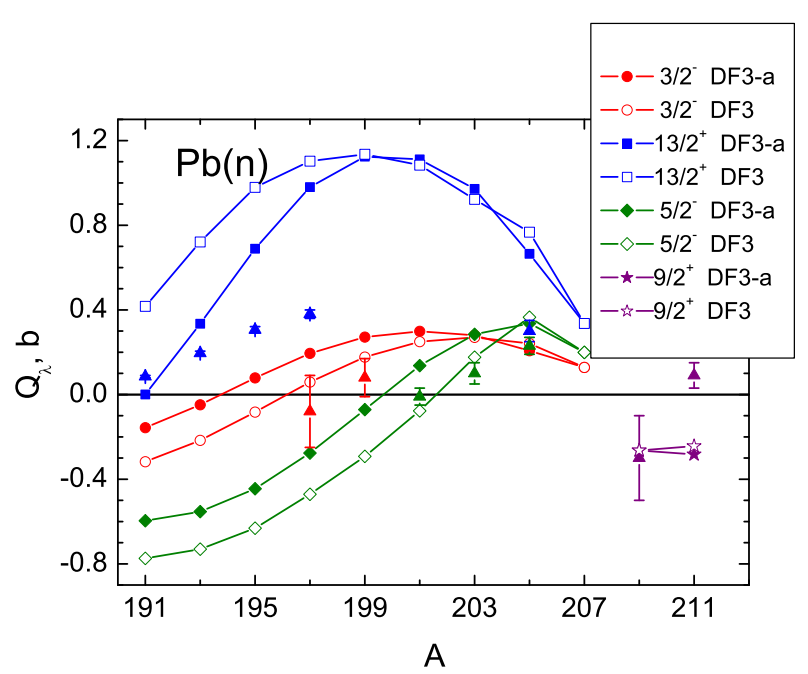

FIG. 5: (Color online) Quadrupole moments of odd lead isotopes with DF3 and DF3-a functionals. Triangles with the bars indicate experimental data [1].

To complete the analysis of quadrupole moments of the odd neighbors of ${ }^{118} \mathrm{Sn}$, we presented in Table \matrix elements $V_{\lambda}$ entering Eq. (10) and quadrupole moments $Q_{\lambda}=\left(u_{\lambda}^{2}-v_{\lambda}^{2}\right) V_{\lambda}$. Note that there is a small difference between these values of $Q_{\lambda}$ and those in Fig. 1 and Table III as in our systematic calculations of quadrupole moments we used in this product for an odd nucleus under consideration the half-sum of $V_{\lambda}$ values in neighboring even nuclei. We see that absolute values of $V_{\lambda}$ for the DF3 functional are almost two times bigger than for the DF3-a functional used by us in the previous article [10]. However, in three cases of four the difference of Bogolyubov factors is opposite and compensates significantly too big values of $V_{\lambda}$ for the DF3 functional. This compensation is almost complete for $7 / 2^{+}$and $11 / 2^{-}$states, the corresponding quadrupole moments for two functionals almost coincide in vicinity of ${ }^{118} \mathrm{Sn}$. This is not the case for the $5 / 2^{+}$state where two Bogolyubov factors almost equal to each other and $V_{\lambda}$ [DF3] almost twice bigger of $\left.V_{\lambda}[\mathrm{DF} 3-\mathrm{a})\right]$. A strong enhancement of the effective field $\hat{V}(r)$ for the DF3 functional in comparison with DF3-a one is shown in Fig. 4. It is seen that the surface maxima of $V_{p}(r)$ and $V_{n}(r)$ functions are almost twice higher in the DF3 case. The higher density of states at the Fermi surface discussed above is causing this effect.

For a deeper understanding of this enhancement effect, 


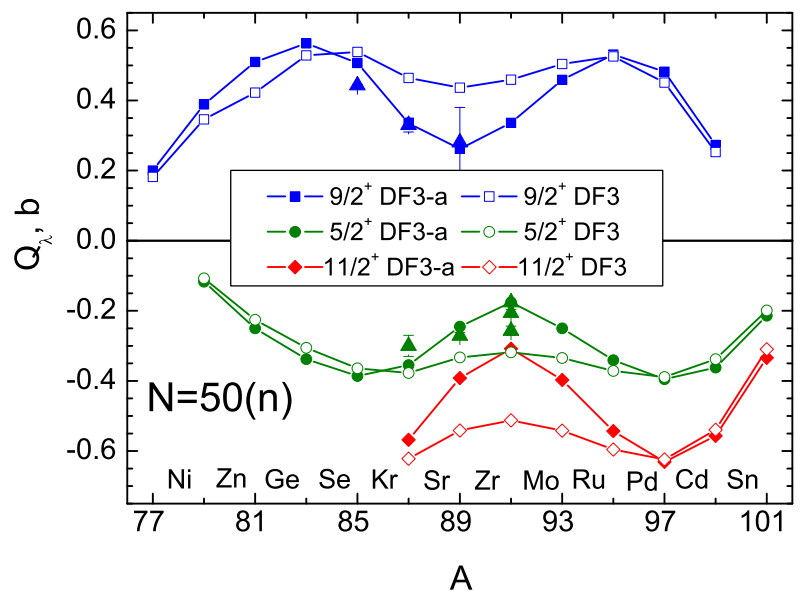

FIG. 6: (Color online) Quadrupole moments of odd-neutron neighbors of even $N=50$ isotones with DF3 and DF3-a functionals. Triangles with bars indicate experimental data [1].

we calculated for the nucleus ${ }^{118} \mathrm{Sn}$ characteristics of the $2_{1}^{+}$state with the DF3 functional, $\omega_{2}=0.913 \mathrm{MeV}$ and $B(E 2)=0.272 \mathrm{e} \mathrm{b}$, and compare them with those calculated previously [10] for the DF3-a functional, $\omega_{2}=$ $1.217 \mathrm{MeV}$ and $B(E 2)=0.172 \mathrm{e} \mathrm{b}$. Note that the DF3a predictions are in reasonable agreement with experimental data, thus the DF3 functional leads to too collective $2_{1}^{+}$state. It explains qualitatively an additional enhancement of the static quadrupole field for the DF3 functional. Indeed, at small external quadrupole field frequency $\omega$, the pole in the $\omega=\omega_{2}$ point term dominates in the effective field,

$$
\hat{V}(\omega)=\frac{2 \omega_{s} \hat{\chi}_{s}}{\omega^{2}-\omega_{s}^{2}}+\hat{V}_{R}(\omega)
$$

where the regular term $\hat{V}_{R}(\omega) \simeq$ const at small $\omega$. The residue of the pole term is $\hat{\chi}_{s}=\left(\hat{V}_{0} \hat{A} \hat{g}_{s}^{+}\right) \hat{g}_{s}, \hat{g}_{s}$ being the amplitude of creating the $s$-th $\left(2_{1}^{+}\right.$in our case) phonon [8]. It can be easily shown that the relation $\chi_{2} \propto B(E 2)$ is valid. If to neglect for an estimation the regular term in Eq. (12), we arrive to $V(\omega=0) \propto B(E 2) / \omega_{2}$. After substituting to this relation the values given above, we find this ratio equal to 0.298 for the DF3 functional and 0.141 for DF3-a. This explains the effect under discussion.

To evaluate the agreement with experiment quantitatively, we calculate the mean theory-experiment difference

$$
\sqrt{\overline{(\delta Q)_{\mathrm{rms}}^{2}}}=\sqrt{\frac{1}{\mathcal{N}} \sum_{i}\left(Q_{i}^{\mathrm{th}}-Q_{i}^{\exp }\right)^{2}},
$$

with obvious notation. For the tin chain under consideration, $\mathcal{N}=11$, we have $\sqrt{\overline{(\delta Q)_{\mathrm{rms}}^{2}}}=0.167 \mathrm{e} \mathrm{b}$ for the DF3 functional and $\sqrt{\overline{(\delta Q)_{\mathrm{rms}}^{2}}}=0.164 \mathrm{e} \mathrm{b}$ for the DF3-a one. Thus, for the DF3-a functional agreement is somewhat better. A remark should be made concerning practical application of Eq. (13). All terms of the sum come with the same weight independent on the experimental error of each measurement. The reason is that, as calculations [10] have shown, our theoretical accuracy for quadrupole moments is not better than $0.1 \mathrm{e} \mathrm{b}$. Practically all the data we use for comparison have better accuracy therefore for our analysis they are equivalent. The nucleus ${ }^{209} \mathrm{~Pb}$ is the only exception where the experimental error is $0.2 \mathrm{e} \mathrm{b}$. To take it into account, we multiply this term with the factor $(0.1 / 0.2)^{2}=1 / 4$. If the table [1] contains several different data on the quadrupole moment under consideration, say 3 , we take into account all of them in the sum of (13) with the weight $1 / 3$.

Let us go now to odd lead nuclei, Fig. 5. We see that again there is a reasonable agreement with experiment for both the functionals with the exception of the intruder state $1 i_{13 / 2}$. For the latter, in the case of the DF3-a functional, a reasonable agreement with the data is found only for two lightest isotopes ${ }^{191,193} \mathrm{~Pb}$. For other four nuclei with known values of $Q\left(1 i_{13 / 2}\right)$ disagreement is significant, the maximal value of $\delta Q=Q^{\text {th }}-Q^{\exp }$ for ${ }^{197} \mathrm{~Pb}$ is $0.60 \mathrm{e} \mathrm{b}$. For the DF3 functional the situation is noticeably worse. Now the rms deviation of the theory from experiment, Eq. (13), is $\sqrt{\overline{\overline{(\delta Q)_{\mathrm{rms}}^{2}}}}[\mathrm{DF} 3-\mathrm{a}]=0.277 \mathrm{e} \mathrm{b}$ and $\sqrt{\overline{(\delta Q)_{\mathrm{rms}}^{2}}}[\mathrm{DF} 3]=0.386 \mathrm{e} \mathrm{b}$. Now the quality of agreement became significantly worse than for tin chain for both functionals but the deterioration is much stronger for the DF3 functional.

In Fig. 6 quadrupole moments are displayed of oddneutron neighbors, $N=50 \pm 1$, of even isotones with $N=50$. In this case, the proton-subsystem is superfluid and the neutron Bogolyubov factor in Eq. (10) is \pm 1 . Agreement with the data is almost perfect for the DF3a functional, $\sqrt{\overline{(\delta Q)_{\mathrm{rms}}^{2}}}[\mathrm{DF} 3-\mathrm{a}]=0.041 \mathrm{e} \mathrm{b}$, and only a little worse for the DF3 functional, $\sqrt{\overline{(\delta Q)_{\mathrm{rms}}^{2}}}[\mathrm{DF} 3]=$ 0.116 e b. For this chain we showed also quadrupole moments of the intruder state $1 h_{11 / 2}$ although none of them was measured. Contrary to tin and lead isotopic chains, none of the functions $Q_{\lambda}(A)$ changes the sign. The situation is similar for odd-neutron neighbors of even isotones with $N=82$ presented in Fig. 7. Again agreement with the data is very good for the DF3-a functional, $\sqrt{\overline{(\delta Q)_{\mathrm{rms}}^{2}}}[\mathrm{DF} 3-\mathrm{a}]=0.093 \mathrm{e} \mathrm{b}$, and again only a little worse for the DF3 functional, $\sqrt{\overline{(\delta Q)_{\mathrm{rms}}^{2}}}[\mathrm{DF} 3]=0.119 \mathrm{e}$ b. In this case, there is one experimental data for the intruder state $1 i_{13 / 2}$ for which the DF3-value practically coincides with experiment.

Table II contains odd-neutron nuclei with known experimental quadrupole moments. We have seen that for each chain considered the DF3-a functional describes data better than the DF3 one. Therefore we present here only predictions of the DF3-a functional. The table is divided in two parts. In the bottom, the intruder states 
$1 h_{11 / 2}$ and $1 i_{13 / 2}$ are collected. The rms deviation of the theoretical predictions from the data calculated with Eq. 13 for all 42 moments in this Table is rather big, $\sqrt{\overline{(\delta Q)_{\mathrm{rms}}^{2}}}[\mathrm{DF} 3-\mathrm{a}]=0.189 \mathrm{e}$ b. For the DF3 functional, the error is bigger, $\sqrt{\overline{\overline{(\delta Q)_{\mathrm{rms}}^{2}}}}[\mathrm{DF} 3]=0.240 \mathrm{e}$ b. Examining the table, we find that the main part of big deviations $\delta Q$ is concentrated in the "intruder" part of the Table containing 15 moments. If to make the calculation (13) only for the latter, we find $\sqrt{\overline{(\delta Q)_{\text {rms }}^{2}}}$ intruder $]=0.269 \mathrm{e}$ b. At last, rather moderate value of the error will occur if we limit ourselves with quadrupole moments of the rest of 27 "normal" states, $\sqrt{\overline{(\delta Q)_{\mathrm{rms}}^{2}}}[$ normal $]=0.125 \mathrm{e} \mathrm{b}$.

In conclusion of this discussion, note that the formalism of developed pairing we use with particle number conservation on average, as is known [22], works worse in the vicinity of magic numbers. For completeness, we include into analysis nuclei ${ }^{205} \mathrm{~Pb}$ and ${ }^{211} \mathrm{~Pb}$ "dangerous" from this point of view. However, in this case it is natural to expect significant errors induced by this approximation.

Let us go to odd-proton nuclei. Quadrupole moments of odd-proton neighbors of even tin isotopes, those of In $(Z=49)$ and $\mathrm{Sb}(\mathrm{Z}=51)$ are displayed in Fig. 8 . The Bogolyubov factor in (10) is now reduced to -1 for the In chain and +1 for the $\mathrm{Sb}$ chain, thus, all difference between predictions of the two functionals we use may come only from different values of the matrix elements $V_{\lambda}$ of the effective field. We see that in the middle part of both chains $\left|Q_{\lambda}\right|$ for the DF3 functional exceeds that for the DF3-a in two times approximately. Remind that the effective field is that in the even Sn core, the same as was used for odd tin isotopes. Now the matrix elements are taken from the proton component $V_{p}(r)$ whereas previously we dealt with the neutron component $V_{n}(r)$. Both were displayed in Fig. 4 and

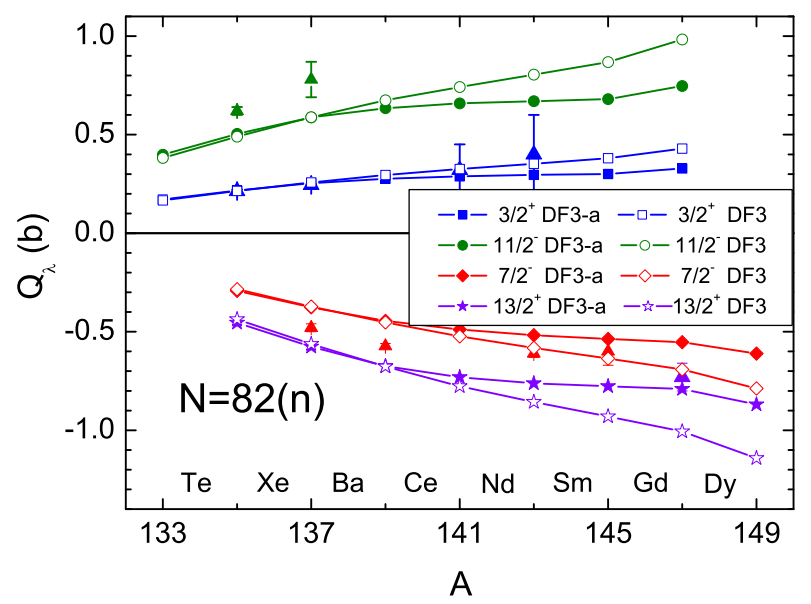

FIG. 7: (Color online) Quadrupole moments of odd-neutron neighbors of even $N=82$ isotones with DF3 and DF3-a functionals. Triangles with the bars indicate experimental data [1].
TABLE II: Quadrupole moments $Q$ (e b) of odd-neutron nuclei in the state $\lambda$. Theoretical values $Q_{\text {th }}$ and differences $\delta Q=Q_{\mathrm{th}}-Q_{\exp }$ are given for the functional DF3-a.

\begin{tabular}{|c|c|c|c|c|}
\hline nucleus & $\lambda$ & $Q_{\exp }$ & $Q_{\mathrm{th}}$ & $\delta Q$ \\
\hline \multirow[t]{2}{*}{${ }^{39} \mathrm{Ca}$} & $1 d_{3 / 2}$ & $0.036(7)$ & +0.040 & 0.004 \\
\hline & & $0.040(6)$ & & 0.000 \\
\hline \multirow[t]{3}{*}{${ }^{41} \mathrm{Ca}$} & $1 f_{7 / 2}$ & $-0.090(2)$ & -0.078 & 0.012 \\
\hline & & $-0.066(2)$ & & -0.012 \\
\hline & & $-0.080(8)$ & & 0.002 \\
\hline${ }^{85} \mathrm{Kr}$ & $1 g_{9 / 2}$ & $+0.443(3)$ & +0.507 & 0.064 \\
\hline${ }^{87} \mathrm{Kr}$ & $2 d_{5 / 2}$ & $-0.30(3)$ & -0.355 & -0.06 \\
\hline${ }^{87} \mathrm{Sr}$ & $1 g_{9 / 2}$ & $+0.33(2)$ & +0.335 & 0.01 \\
\hline${ }^{89} \mathrm{Sr}$ & $2 d_{5 / 2}$ & $-0.271(9)$ & -0.245 & -0.026 \\
\hline${ }^{89} \mathrm{Zr}$ & $1 g_{9 / 2}$ & $+0.28(10)$ & +0.262 & -0.02 \\
\hline \multirow[t]{3}{*}{${ }^{91} \mathrm{Zr}$} & $2 d_{5 / 2}$ & $-0.176(3)$ & -0.195 & -0.019 \\
\hline & & $(-) 0.257(13)$ & & 0.062 \\
\hline & & $-0.206(10)$ & & 0.011 \\
\hline${ }^{109} \mathrm{Sn}$ & $2 d_{5 / 2}$ & $+0.31(10)$ & +0.250 & -0.06 \\
\hline${ }^{111} \mathrm{Sn}$ & $1 g_{7 / 2}$ & $+0.18(9)$ & +0.029 & -0.13 \\
\hline${ }^{115} \mathrm{Sn}$ & $1 g_{7 / 2}^{*}$ & $0.26(3)$ & +0.377 & 0.12 \\
\hline \multirow[t]{3}{*}{${ }^{119} \mathrm{Sn}$} & $2 d_{3 / 2}^{*}$ & $0.094(11)$ & -0.035 & -0.129 \\
\hline & & $-0.065(5)$ & & 0.030 \\
\hline & & $-0.061(3)$ & & 0.026 \\
\hline${ }^{121} \mathrm{Sn}$ & $2 d_{3 / 2}$ & $-0.02(2)$ & +0.063 & 0.08 \\
\hline${ }^{135} \mathrm{Xe}$ & $2 d_{3 / 2}$ & $+0.214(7)$ & +0.217 & 0.003 \\
\hline${ }^{137} \mathrm{Xe}$ & $2 f_{7 / 2}$ & $-0.48(2)$ & -0.376 & 0.10 \\
\hline${ }^{137} \mathrm{Ba}$ & $2 d_{3 / 2}$ & $+0.245(4)$ & +0.254 & 0.009 \\
\hline${ }^{139} \mathrm{Ba}$ & $2 f_{7 / 2}$ & $-0.573(13)$ & -0.445 & 0.128 \\
\hline${ }^{141} \mathrm{Nd}$ & $2 d_{3 / 2}$ & $+0.32(13)$ & +0.289 & -0.03 \\
\hline${ }^{143} \mathrm{Nd}$ & $2 f_{7 / 2}$ & $-0.61(2)$ & -0.518 & 0.09 \\
\hline${ }^{143} \mathrm{Sm}$ & $2 d_{3 / 2}$ & $+0.4(2)$ & +0.296 & 0.1 \\
\hline${ }^{145} \mathrm{Sm}$ & $2 f_{7 / 2}$ & $-0.60(7)$ & -0.537 & 0.06 \\
\hline${ }^{197} \mathrm{~Pb}$ & $3 p_{3 / 2}$ & $-0.08(17)$ & +0.195 & 0.27 \\
\hline${ }^{199} \mathrm{~Pb}$ & $3 p_{3 / 2}$ & $+0.08(9)$ & +0.272 & 0.19 \\
\hline${ }^{201} \mathrm{~Pb}$ & $2 f_{5 / 2}$ & $-0.01(4)$ & +0.137 & 0.15 \\
\hline${ }^{203} \mathrm{~Pb}$ & $2 f_{5 / 2}$ & $+0.10(5)$ & +0.284 & 0.18 \\
\hline${ }^{205} \mathrm{~Pb}$ & $2 f_{5 / 2}$ & $+0.23(4)$ & +0.336 & 0.09 \\
\hline${ }^{209} \mathrm{~Pb}$ & $2 g_{9 / 2}$ & $-0.3(2)$ & -0.264 & 0.1 \\
\hline${ }^{211} \mathrm{~Pb}$ & $2 g_{9 / 2}$ & $+0.09(6)$ & -0.283 & -0.37 \\
\hline \multirow[t]{2}{*}{${ }^{113} \mathrm{Sn}$} & $\overline{11 h_{11 / 2}^{*}}$ & $\overline{0.41(4)}$ & $\overline{-0.776}$ & -0.37 \\
\hline & & $0.48(5)$ & & -0.30 \\
\hline${ }^{115} \mathrm{Sn}$ & $1 h_{11 / 2}^{*}$ & $0.38(6)$ & -0.703 & -0.32 \\
\hline${ }^{117} \mathrm{Sn}$ & $1 h_{11 / 2}^{*}$ & $-0.42(5)$ & -0.593 & -0.17 \\
\hline${ }^{119} \mathrm{Sn}$ & $1 h_{11 / 2}^{*}$ & $0.21(2)$ & -0.469 & -0.25 \\
\hline${ }^{121} \mathrm{Sn}$ & $1 h_{11 / 2}^{*}$ & $-0.14(3)$ & -0.293 & -0.15 \\
\hline${ }^{123} \mathrm{Sn}$ & $1 h_{11 / 2}$ & $+0.03(4)$ & -0.123 & -0.15 \\
\hline${ }^{125} \mathrm{Sn}$ & $1 h_{11 / 2}$ & $+0.1(2)$ & +0.039 & -0.1 \\
\hline${ }^{135} \mathrm{Xe}$ & $1 h_{11 / 2}^{*}$ & $+0.62(2)$ & +0.504 & 0.12 \\
\hline${ }^{137} \mathrm{Ba}$ & $1 h_{11 / 2}^{*}$ & $+0.78(9)$ & +0.588 & -0.19 \\
\hline${ }^{147} \mathrm{Gd}$ & $1 i_{13 / 2}^{*}$ & $-0.73(7)$ & -0.791 & -0.06 \\
\hline${ }^{191} \mathrm{~Pb}$ & $1 i_{13 / 2}^{*}$ & $+0.085(5)$ & +0.0004 & -0.085 \\
\hline${ }^{193} \mathrm{~Pb}$ & $1 i_{13 / 2}^{*}$ & $+0.195(10)$ & +0.335 & 0.140 \\
\hline${ }^{195} \mathrm{~Pb}$ & $1 i_{13 / 2}^{*}$ & $+0.306(15)$ & +0.689 & 0.383 \\
\hline${ }^{197} \mathrm{~Pb}$ & $1 i_{13 / 2}^{*}$ & $+0.38(2)$ & +0.980 & 0.60 \\
\hline${ }^{205} \mathrm{~Pb}$ & $1 i_{13 / 2}^{*}$ & $0.30(5)$ & +0.665 & 0.37 \\
\hline
\end{tabular}

we have seen that both of them are approximately two times bigger for the DF3 functional. This was caused 
by a too strong collective $2_{1}^{+}$state for this functional. For tin isotopes, this deficiency was partially compensated with different values of the Bogolyubov factor, see Tab. [1] Now we see the effect of the effective field directly, without any distortions. In the case of the DF3-a functional, the rms deviation is worse than for tin isotopes but moderately, $\sqrt{\overline{(\delta Q)_{\mathrm{rms}}^{2}}}[\mathrm{DF} 3-\mathrm{a}]=0.249$ e b. As to the DF3 functional, the error grows drastically, $\sqrt{\overline{(\delta Q)_{\mathrm{rms}}^{2}}}[\mathrm{DF} 3]=0.688 \mathrm{e} \mathrm{b}$. There is a feeling that for the DF3-a functional the effective field $V_{p}(r)$ is also too strong and the agreement will become better if it will be reduced by $20-30 \%$. Within the mean field approach we use it can be achieved only with such variation of the central part of the EDF, common to DF3 and DF3-a, which changes the LM amplitude $\mathcal{F}_{n p}$.

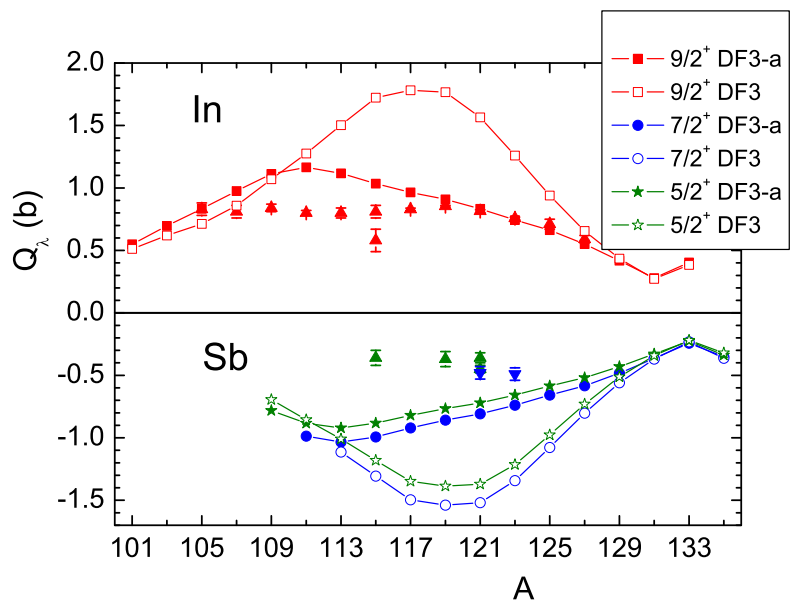

FIG. 8: (Color online) Quadrupole moments of odd-proton neighbors of even tin isotopes with DF3 and DF3-a functionals. Triangles with the bars indicate experimental data [1].

Quadrupole moments of the odd neighbors of even lead isotopes are presented in Fig. 9. We see that here the difference between DF3 and DF3-a functionals is not big, especially in the right half of the figure where some experimental data exist. Unfortunately, their number is limited, only one for $\mathrm{Tl}$ and five for $\mathrm{Bi}$. In the last case, there are two sets of the data [1]. Both the calculations agree with one of them and disagree with the other. In such a situation, it is not worth to estimate the average disagreement numerically as it was made above.

Let us go to the chain of odd-proton with magic neutron number $N=50$, eleven nuclei from ${ }^{79} \mathrm{Cu}$ till ${ }^{99} \mathrm{In}$, see Fig. 10. Note that all of them, except ${ }^{89} \mathrm{Y}$, are $\beta$ unstable, many being far beyond the $\beta$-stability valley. Here we deal with proton pairing, and the Bogolyubov factor in Eq. (10) varies strongly from one nucleus to another and depends on the state $\lambda$. However, for this chain we obtain rather close predictions of both functionals used. In this case, the difference between both functionals is not as strong as in the case of the $1 h_{11 / 2}$ intruder state in the tin isotopes. Unfortunately, the ex-

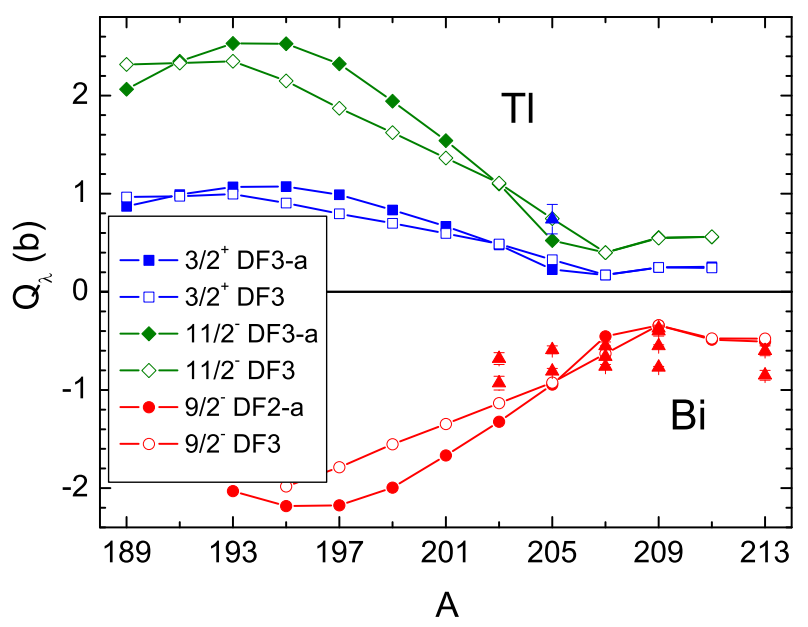

FIG. 9: (Color online) Quadrupole moments of odd-proton neighbors of even lead isotopes with DF3 and DF3-a functionals. Triangles with the bars indicate experimental data [1].

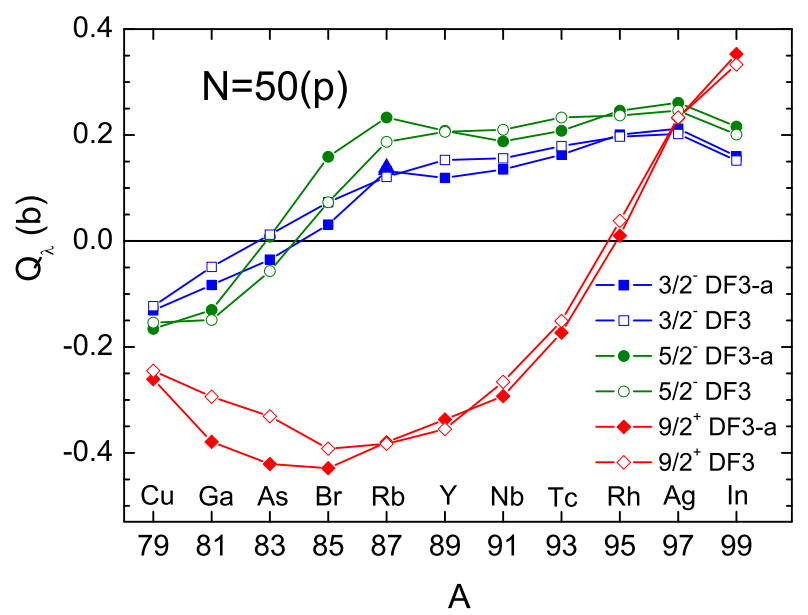

FIG. 10: (Color online) Quadrupole moments of odd-proton $N=50$ isotones with DF3 and DF3-a functionals. Triangles with the bars indicate experimental data 1].

perimental quadrupole moment is known for one nucleus only, ${ }^{87} \mathrm{Rb}$. For this, both theoretical predictions practically coincide with the experimental values.

Quadrupole moments of the chain of odd-proton with magic neutron number $N=82$ are displayed in Fig. 11. Here we deal with seven nuclei, from ${ }^{135} \mathrm{I}$ till ${ }^{147} \mathrm{~Tb}$. Among them there are two stable ones, ${ }^{139}$ La and ${ }^{141} \mathrm{Pr}$, the others are $\beta$-unstable, but only ${ }^{135}$ I lives hours, the rest, days or years. For this chain, four quadrupole moments are known. For the DF3-a functional, disagreement with the data doesn't exceed $0.1 \mathrm{e} \mathrm{b.} \mathrm{For} \mathrm{the}$ DF3 one, disagreement is greater but not significantly. Comparison between predictions of the two functionals shows very close results for the $2 d_{3 / 2}$ and $2 d_{5 / 2}$ states. It demonstrates close results for the effective field $V_{p}(r)$. A 


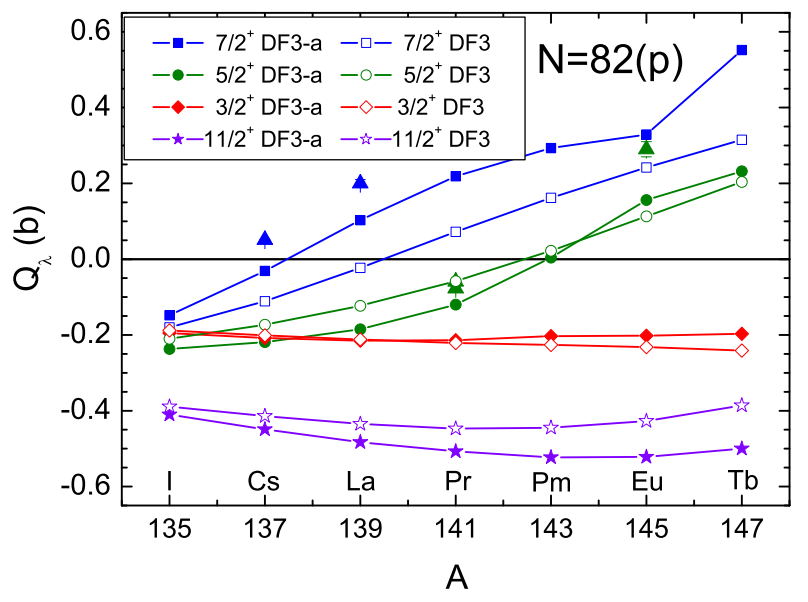

FIG. 11: (Color online) Quadrupole moments of odd-proton $N=82$ isotones with DF3 and DF3-a functionals. Triangles with the bars indicate experimental data [1].

stronger difference for the $1 g_{7 / 2}$ state is due to the difference of the Bogolyubov factors in Eq. (10) similar to that we have seen for the neutron $1 g_{7 / 2}$ state, see Fig. 3. It is worth to mention that the difference between two predictions for the proton intruder $1 i_{13 / 2}$ state is significantly less than for the neutron one in the lead isotopes, Fig. 5. More exactly, for neutrons the difference was strong for lead isotopes lighter of ${ }^{200} \mathrm{~Pb}$ and rather small, for heavier ones. Such behavior is explained with dynamics of variation of the spin-orbit density with filling the neutron shell which strongly influences the position of high $j$-levels.

All quadrupole moments of odd-proton nuclei with known experimental values are collected in Table [II] Just as for neutrons, we concentrated on the DF3-a functional which turned out to be more successful than the DF3 one. For the DF3 functional, the average error for protons is too big, $\sqrt{\overline{(\delta Q)_{\mathrm{rms}}^{2}}}[\mathrm{DF} 3]=0.589 \mathrm{e} \mathrm{b}$, whereas $\sqrt{\overline{(\delta Q)_{\mathrm{rms}}^{2}}}[\mathrm{DF} 3-\mathrm{a}]=0.254 \mathrm{e}$ b. However, for the DF3a functional in average protons are also described worse than neutrons. The main contribution to this deviation comes from In and $\mathrm{Sb}$ isotopes, odd neighbors of even tin nuclei. As it was written above, it is the result of too strong quadrupole field $V_{n, p}(r)$ even for DF3-a functional, not only for the DF3 one. For neutrons this drawback is partially hidden with multiplying by the Bogolyubov factor, see Table I but for protons it appears to the full extent. In many cases, we feel, the main reason of disagreement is the neglect of corrections from the particlephonon coupling. Although in semi-magic nuclei this coupling is relatively small and can be accounted for with perturbation theory, sometimes these corrections could be noticeable. E.g. for the excited $3 / 2^{+}$state in ${ }^{205} \mathrm{Tl}$ a strong admixture of the $\left(2^{+} \otimes 1 / 2^{+}\right)_{3 / 2}$ state may be expected. It could explain the very strong deviation $\delta Q$ in this case.

\section{CONCLUSIONS}

Quadrupole moments of odd neighbors of semi-magic lead and tin isotopes and $N=50, N=82$ isotones are calculated within the self-consistent TFFS on the base of the Energy Density Functional by Fayans et al. Two sets of the EDF parameters are used fixed previously. Namely, the functional DF3 [6, 7] is used and its modification DF3-a [11]. The latter is obtained with a small vari-

TABLE III: Quadrupole moments $Q$ (b) of odd-proton nuclei in the state $\lambda$. Theoretical values $Q_{\mathrm{th}}$ and differences $\delta Q=$ $Q_{\mathrm{th}}-Q_{\exp }$ are given for the functional DF3-a.

\begin{tabular}{|c|c|c|c|c|}
\hline $\begin{array}{l}\text { nucl. } \\
\text { nut }\end{array}$ & $\overline{\bar{\lambda}}$ & $\overline{\overline{Q_{\exp }}}$ & 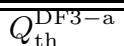 & $\overline{\bar{\delta} \delta Q(\mathrm{DF} 3-\mathrm{a})}$ \\
\hline${ }^{39} \mathrm{~K}$ & $1 d_{3 / 2}$ & $0.0585(6)$ & 0.069 & 0.010 \\
\hline \multirow[t]{3}{*}{${ }^{41} \mathrm{Sc}$} & $1 f_{7 / 2}$ & $-0.156(3)$ & -0.139 & 0.017 \\
\hline & & $0.120(6)$ & & -0.019 \\
\hline & & $0.168(8)$ & & 0.029 \\
\hline \multirow[t]{2}{*}{${ }^{87} \mathrm{Rb}$} & $2 p_{3 / 2}$ & $+0.134(1)$ & +0.132 & -0.002 \\
\hline & & $+0.138(1)$ & & -0.006 \\
\hline${ }^{105} \mathrm{In}$ & $1 g_{9 / 2}$ & $+0.83(5)$ & +0.833 & 0.00 \\
\hline${ }^{107} \mathrm{In}$ & $1 g_{9 / 2}$ & $+0.81(5)$ & +0.976 & 0.17 \\
\hline${ }^{109} \mathrm{In}$ & $1 g_{9 / 2}$ & $+0.84(3)$ & +1.113 & 0.27 \\
\hline${ }^{111} \mathrm{In}$ & $1 g_{9 / 2}$ & $+0.80(2)$ & +1.165 & 0.36 \\
\hline${ }^{113} \mathrm{In}$ & $1 g_{9 / 2}$ & $+0.80(4)$ & +1.117 & 0.32 \\
\hline \multirow[t]{2}{*}{${ }^{115} \mathrm{In}$} & $1 g_{9 / 2}$ & $+0.81(5)$ & +1.034 & 0.22 \\
\hline & & $0.58(9)$ & & 0.45 \\
\hline${ }^{117} \mathrm{In}$ & $1 g_{9 / 2}$ & $+0.829(10)$ & +0.965 & 0.136 \\
\hline${ }^{119} \mathrm{In}$ & $1 g_{9 / 2}$ & $+0.854(7)$ & +0.909 & 0.055 \\
\hline${ }^{121} \mathrm{In}$ & $1 g_{9 / 2}$ & $+0.814(11)$ & +0.833 & 0.019 \\
\hline${ }^{123} \mathrm{In}$ & $1 g_{9 / 2}$ & $+0.757(9)$ & +0.743 & -0.014 \\
\hline${ }^{125} \mathrm{In}$ & $1 g_{9 / 2}$ & $+0.71(4)$ & +0.663 & -0.05 \\
\hline${ }^{127} \mathrm{In}$ & $1 g_{9 / 2}$ & $+0.59(3)$ & +0.550 & -0.04 \\
\hline${ }^{115} \mathrm{Sb}$ & $2 d_{5 / 2}$ & $-0.36(6)$ & -0.882 & -0.52 \\
\hline${ }^{119} \mathrm{Sb}$ & $2 d_{5 / 2}$ & $-0.37(6)$ & -0.766 & -0.40 \\
\hline \multirow[t]{2}{*}{${ }^{121} \mathrm{Sb}$} & $2 d_{5 / 2}$ & $-0.36(4)$ & -0.721 & -0.36 \\
\hline & & $-0.45(3)$ & & -0.27 \\
\hline${ }^{123} \mathrm{Sb}$ & $1 g_{7 / 2}$ & $-0.49(5)$ & -0.739 & -0.25 \\
\hline${ }^{137} \mathrm{Cs}$ & $1 g_{7 / 2}$ & $+0.051(1)$ & -0.031 & -0.080 \\
\hline${ }^{139} \mathrm{La}$ & $1 g_{7 / 2}$ & $+0.20(1)$ & +0.103 & -0.10 \\
\hline \multirow[t]{2}{*}{${ }^{141} \mathrm{Pr}$} & $2 d_{5 / 2}$ & $-0.077(6)$ & -0.120 & -0.043 \\
\hline & & $-0.059(4)$ & & -0.061 \\
\hline${ }^{145} \mathrm{Eu}$ & $2 d_{5 / 2}$ & $+0.29(2)$ & +0.156 & -0.13 \\
\hline${ }^{205} \mathrm{Tl}$ & $3 d_{3 / 2}^{*}$ & $+0.74(15)$ & +0.227 & -0.51 \\
\hline \multirow[t]{2}{*}{${ }^{203} \mathrm{Bi}$} & $1 h_{9 / 2}$ & $-0.93(7)$ & -1.323 & -0.39 \\
\hline & & $-0.68(6)$ & & -0.64 \\
\hline \multirow[t]{2}{*}{${ }^{205} \mathrm{Bi}$} & $1 h_{9 / 2}$ & $-0.81(3)$ & -0.945 & -0.14 \\
\hline & & $-0.59(4)$ & & -0.36 \\
\hline \multirow[t]{3}{*}{${ }^{207} \mathrm{Bi}$} & $1 h_{9 / 2}$ & $-0.76(2)$ & -0.454 & 0.31 \\
\hline & & $-0.55(4)$ & & 0.10 \\
\hline & & $-0.60(11)$ & & 0.15 \\
\hline \multirow[t]{6}{*}{${ }^{209} \mathrm{Bi}$} & $1 h_{9 / 2}$ & $-0.516(15)$ & -0.342 & 0.18 \\
\hline & & $-0.37(3)$ & & 0.03 \\
\hline & & $-0.55(1)$ & & 0.21 \\
\hline & & $-0.77(1)$ & & 0.43 \\
\hline & & $-0.40(5)$ & & 0.06 \\
\hline & & $-0.39(3)$ & & 0.05 \\
\hline \multirow[t]{2}{*}{${ }^{213} \mathrm{Bi}$} & $1 h_{9 / 2}$ & $-0.83(5)$ & -0.508 & 0.32 \\
\hline & & $-0.60(5)$ & & 0.09 \\
\hline
\end{tabular}


ation in the initial functional DF3 of the spin-orbit terms and rather strong, of the effective tensor term. The latter influences the spin-orbit splitting in nuclei with partially filled spin-orbit doublets where the spin-orbit density $\rho_{s l}$ is non-zero. Due to different spin-orbit splitting, the position of high $j$-levels and all the single-particle spectrum in the vicinity of Fermi level are often different for two functionals under consideration. The complete set of the QRPA-like TFFS equations for the effective field is solved in a self-consistent way.

Recently, we used this method [10] to calculate characteristics of the $2_{1}^{+}$states in even tin and lead isotopes with the use of the DF3-a functional. In this work, quadrupole moments were calculated as well of odd tin and lead isotopes and odd-proton neighbors, In and Sb isotopes in the first case and $\mathrm{Tl}$ and $\mathrm{Bi}$ isotopes in the second one. Strong sensitivity of quadrupole moments of odd-neutron tin and lead isotopes to the single-particle spectrum in the vicinity of the Fermi surface was found in [10] due to the Bogolyubov factor $u_{\lambda}^{2}-v_{\lambda}^{2}=\left(\varepsilon_{\lambda}-\mu\right) / E_{\lambda}$.

To examine this effect in more detail, in the present paper we calculated quadrupole moments with the DF3 functional. These calculations confirmed the observation of Ref. [10]. In addition, it was found that the static quadrupole effective field $V(r)$, the main ingredient of the formula for the quadrupole moment $Q_{\lambda}$, also can be significantly different for these two functionals, again due to different level structure in the vicinity of the Fermi level. This occurs in the middle part of the tin isotopes where the DF3 effective field approximately two times stronger than the DF3-a one. The latter looks more realistic as follows from the analysis of the characteristics $\omega_{2}$ and BE2 in even tin isotopes. 23] For odd tin isotopes, a too strong effective field is hidden partially with smaller values of the Bogolyubov factor, but the situation is worse for odd-proton neighbors, In and $\mathrm{Sb}$ isotopes, where the DF3 functional leads to a strong disagreement with experiment. For other chains considered the difference between the predictions of both functionals is less, but on the whole, the DF3-a functional describes quadrupole moments better than the DF3 one.

For the DF3-a functional, the agreement, on average, can be considered as reasonable. For 42 quadrupole moments of odd-neutron nuclei, the average disagreement between theory and experiment is not so small, $\sqrt{\overline{(\delta Q)_{\mathrm{rms}}^{2}}}=0.189$ e b. However, it is concentrated mainly in 15 intruder states for which we have $\sqrt{\overline{(\delta Q)_{\mathrm{rms}}^{2}}}$ [intruder $]=0.269$ e b. For the rest of 27 "normal" states, the disagreement is rather moderate $\sqrt{\overline{(\delta Q)_{\mathrm{rms}}^{2}}}[$ normal $]=0.125 \mathrm{e} \mathrm{b}$.

For protons, agreement is worse. Leaving aside specific reasons in several cases, we see the main source of disagreement here, just as for neutrons, in neglecting the effects of the coupling of single-particle degrees of freedom with phonons, see e.g. [3, 4, 21].

The comparison between two functionals favors DF3a which has a rather strong effective tensor force. For further improvements, phonon contributions should be taken into account which would pave the way to extend the present investigation to nuclei with both non-magic proton and neutron subsystems.

\section{ACKNOWLEDGMENT}

The work was partly supported by the DFG and RFBR Grants Nos.436RUS113/994/0-1 and 09-02-91352NNIOa, by the Grants NSh-7235.2010.2 and 2.1.1/4540 of the Russian Ministry for Science and Education, and by the RFBR grants 09-02-01284-a, 11-02-00467-a. Four of us, S. T., S. Ka., E. S., and D. V., are grateful to the Institut für Kernphysik, Forschungszentrum Jülich for its hospitality.
[1] N. J. Stone, Atomic Data Nuclear Data Table 90, (2005) 75.

[2] P. Vingerhoets, K. T. Flanagan, M. Avgoulea, et. al., Phys. Rev. C 82, 064311 (2010).

[3] I. N. Borzov, E. E. Saperstein, S. V. Tolokonnikov, Phys. At. Nucl. 71, (2008) 469.

[4] I. N. Borzov, E. E. Saperstein, S. V. Tolokonnikov, G. Neyens, and N. Severijns, Eur. Phys. J. A 45, 159 (2010).

[5] A. V. Smirnov, S. V. Tolokonnikov, and S. A. Fayans, Sov. J. Nucl. Phys. 48, 995 (1988).

[6] S. A. Fayans, JETP Letters 68, 169 (1998).

[7] S. A. Fayans, S. V. Tolokonnikov, E. L. Trykov, and D. Zawischa, Nucl. Phys. A676, 49 (2000).

[8] A. B. Migdal, Theory of finite Fermi systems and applications to atomic nuclei (Wiley, New York, 1967).

[9] M. Honma, T. Otsuka, B. A. Brown, T. Mizusaki, Phys. Rev. C 69, (2004) 034335.

[10] S. V. Tolokonnikov, S. Kamerdzhiev, D. Voytenkov, S.
Krewald, and E. E. Saperstein, arXiv:1107.2432 v2[nuclth], Phys. Rev. C, to be published.

[11] S. V. Tolokonnikov and E. E. Saperstein, Phys. Atom. Nucl. 73, 1684 (2010).

[12] V. A. Khodel and E. E. Saperstein, Phys. Rep. 92, 183 (1982).

[13] V. G. Soloviev, Theory of Complex Niclei, (Oxford: Pergamon Press, 1976).

[14] B. A. Brown, T. Duguet, T. Otsuka, et al., Phys. Rev. C 74, 061303(R) (2006).

[15] M. Bender, K. Bennaceur, T. Duguet, et al., Phys. Rev. C 80, 064302 (2009).

[16] S. S. Pankratov, M. V. Zverev, M. Baldo, U. Lombardo, and E. E. Saperstein, Phys. Rev. C 84, 014321 (2011).

[17] M. Baldo, U. Lombardo, E. E. Saperstein, M. V. Zverev, Phys. Rep. 391 261, (2004).

[18] W. Kohn and L. J. Sham, Phys. Rev. 140, A 1133 (1965).

[19] L. N. Oliveira, E. K. U. Gross, and W. Kohn, Phys. Rev. 
Lett. 60, 2430 (1988).

[20] A. Bohr and B. R. Mottelson, Nuclear Structure (Benjamin, New York, Amsterdam, 1969.), Vol. 1.

[21] S. P. Kamerdzhiev, A. V. Avdeenkov and D. A. Voitenkov, Phys. Atom. Nucl. 73, to be published (2011).
[22] Abhishek Mukherjee, Y. Alhassid, and G. F. Bertsch, Phys. Rev. C 83, 014319 (2011).

[23] In fact, such analysis was made for ${ }^{118} \mathrm{Sn}$ nucleus. 